\title{
Research on Time-Frequency Domain Acquisition Algorithm of Parallel Combinatory Spread Spectrum System Based on FFT
}

\author{
L.L. Guo \\ College of Information and Communication Engineering \\ Harbin Engineering University \\ Harbin, China \\ Science and Technology on Information Transmission and \\ Dissemination in Communication Networks Laboratory \\ Shijiazhuang, China
}

\section{S. Chen}

College of Information and Communication Engineering Harbin Engineering University

Harbin 150001, China

\begin{abstract}
In this paper, an acquisition algorithm is discussed in parallel combinatory spread spectrum (PCSS) system. The algorithm model is first built. Then, an improved algorithm utilizing multiple correlation peak accumulation under low SNR is proposed. The simulation results show that Doppler offset and PN code-phase delay can be obtained by using the proposed acquisition algorithms. The simulation results also represent that a good performance of detection probability can be obtained by adjusting the number of PN code period in low SNR.
\end{abstract} SNR

Keywords-PCSS; FFT; time-frequency domain acquisition; low

\section{INTRODUCTION}

Parallel combinatory spread spectrum (PCSS) system is proposed on the basis of $\mathrm{M}$-ary and $\mathrm{M}_{\mathrm{b}}$-ary spread spectrum, it has been attracted more and more attentions because of its advantages of high efficiency and security property in recent years [1, 2]. At present, the studies of PCSS focus on modulation [3, 4], data-combinatory sequences mapping algorithm [5, 6], and integrated with other technologies [7, 8], but fewer studies on synchronization technology. Because the transmission of PCSS are combinatory sequences, while the receiver contains multichannel de-spreading correlators, so it is not only complexity and computation of the system is increased, but also cross-interference between the PN sequences is caused. Several commonly acquisition algorithms are applied to PCSS system in article [9] and the FFT algorithm verified by simulation analysis is more suitable than other algorithms in terms of acquisition time and complexity. In PCSS system, the acquisition process consist of combinatory sequences and carrier waves, however, the combinatory sequences acquisition only studied in article [9]. This paper combines FFT algorithm with features of PCSS for further study.

\author{
Z.G. Sun \\ College of Information and Communication Engineering \\ Harbin Engineering University \\ Harbin, China \\ Science and Technology on Information Transmission and \\ Dissemination in Communication Networks Laboratory \\ Shijiazhuang, China
}

\author{
B.C. Wang, X.Y. Ning \\ College of Information and Communication Engineering \\ Harbin Engineering University \\ Harbin, China
}

\section{PARALLEL COMBINATORY SPREAD SPECTRUM SySTEM}

At the transmitter of PCSS system, a total of $M$ orthogonal pseudo-noise (PN) sequences are assigned for one user. According to the mapping algorithm, $r$ sequences with multiplying factor $(+1,-1)$ are transmitted in parallel correspond to each $k$ data bits. The information bits transmitted at one time per period of orthogonal PN sequence are

$$
k=r+\left\lfloor\log _{2}\left(\mathrm{C}_{M}^{r}\right)\right\rfloor
$$

The transmitter of PCSS system is shown in fig. 1 .

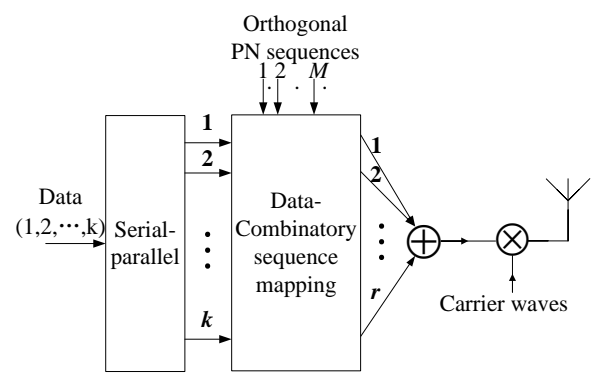

FIGURE I. THE TRANSMITTER OF PCSS SYSTEM

These $\mathrm{k}$ bits data is represented by $\left\{d_{1}, d_{2}, \cdots, d_{k}\right\}$ which should be converted from serial data bits into parallel ones first. Then the data is sent to data-combinatory sequences mapper, and the serial numbers and states of $r$ sequences are chosen from $M$ PN sequences based on $r$-combinatory theory $[4$, 6]. After $r$ sequences are added up in constant amplitude. The combinatory sequences are shown as below 


$$
D(t)=\sum_{j=1}^{r} q_{l j} P N_{l j}(t)
$$

where

$$
q_{l j} \in(-1,+1),(l=1,2, \cdots M, j=1,2, \cdots r)
$$

represents

the state of $\mathrm{PN}$ sequence.

This signal is transmitted in the channel after carrier modulation and the channel is assumed as ideal synchronous additive white Gaussian noise. The received signal is

$$
s(t)=\sqrt{2 P} D(t) \cos \left(2 \pi f_{c} t+\theta\right)
$$

where $P$ is the power of carrier waves, $n(t)$ is the white guassion noise.

The receiver of PCSS system is described in fig. 2 .

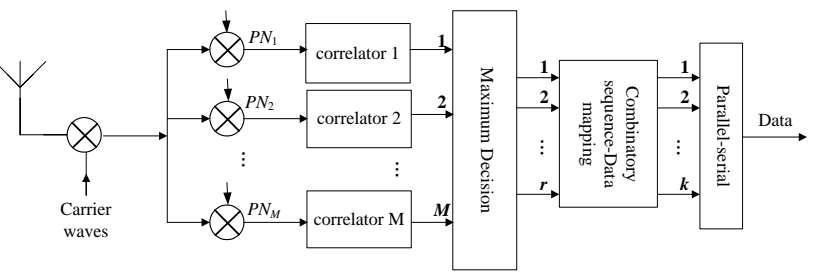

FIGURE II. THE RECEIVER OF PCSS SYSTEM

$M$ de-spreading correlators are contained in the receiver of PCSS system. The output results are given below

$$
Z_{l}(t)=\left\{\begin{array}{l}
q_{l j} P T+N_{l}(t) \quad l=l j \\
N_{l}(t) \quad l \neq l j
\end{array}\right.
$$

where $T$ is period of a PN sequence, and $N_{l}(t)$ represents the output value of guassion white noise after de-spreading. Each output of $Z(t)$ is compared, $r$ sequences which have larger values are selected. Then the combinatory number is sent to combinatory sequences-data mapper. Finally, the output data are obtained through the parallel to serial converter [7].

\section{TIME-FREQUENCY ACQUISITION ALGORITHM BASED ON FFT}

\section{A. The Principle of Acquisition Algorithm}

Traditional DSSS acquisition methods include serial search technique, matched filter technique, and parallel search technique using FFT [10, 11]. As is shown in fig. 2, the receiver complexity of PCSS system is $M$ times than DSSS, so the computation of acquisition process increases $M$ times. Therefore, a fast acquisition method based on FFT proposed to PCSS system, which the principle is given by

$$
R(m)=x(-n) \otimes h(n)=\sum_{n=0}^{N-1} x(n+m) h(n)=\frac{1}{N} \operatorname{IFFT}\left[X^{*}(k) H(k)\right]
$$

where $\otimes$ represents circuit convolution, $X^{*}(k)$ is the complex conjugate of the spectrum of $x(n+m), H(k)$ is the spectrum of $h(n)$, and $N$ is the length of PN sequence. As is observed in eqn (5), the computation of time domain correlation function is proportional to $N^{2}$. However, much computing time can be saved when the time domain convolution is calculated via the frequency domain.

The whole scheme is depicted in fig. 3: In the sweeping range, a frequency $f^{\prime}$ is first generated by local carrier oscillator, and the received IF signal is down converted into baseband signal. After $M \times N$ order correlation matrix is obtained through the FFT acquisition method. Then the larger $r$ of $M$ rows are non-coherent accumulated. Finally, all accumulation results are compared against the threshold set by our technique. If any result exceeds the threshold, the acquisition of signal is alleged successfully. If not, the acquisition process will repeat continuously.

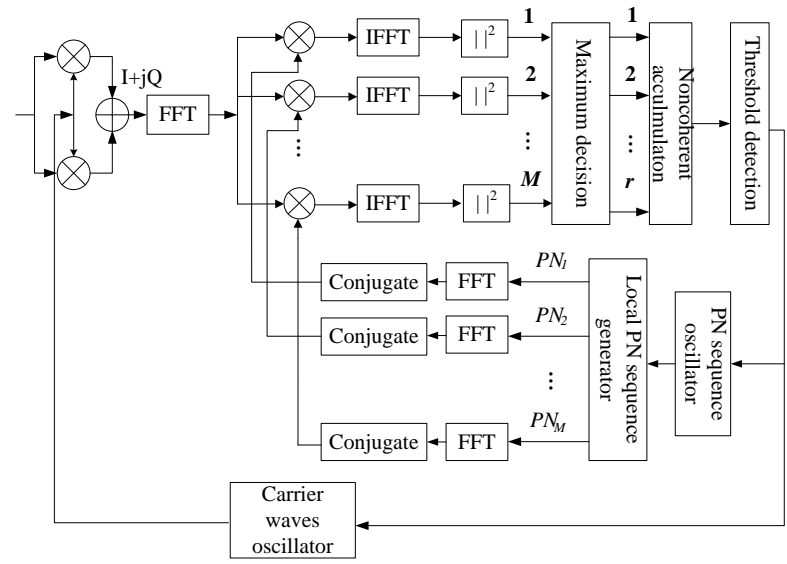

FIGURE III. TIME-FREQUENCY DOMAIN ACQUISITION SCHEME BASED ON FFT

\section{B. Theoretical Analysis on Suppressing Doppler Offset}

The IF signal phase $\theta=0$ is assumed and the high-frequency component is ignored, the baseband signal is obtained through a down-converter

$$
r\left(i T_{s}\right)=D\left(i T_{s}-\tau\right) \exp \left(\mathrm{j} 2 \pi f_{d} i T_{s}\right)
$$

where $D\left(i T_{s}\right)$ is the received combinatory sequences, $\tau$ is the PN code-phase delay, $f_{c}$ is the carrier frequency, $f_{d}$ is the Doppler offset, $T_{s}$ is the sampling period, $\theta$ is the IF signal phase. Then the $l$ de-spreading correlator normalized frequency response as below

$$
E_{l}\left(f_{d}\right)=\left\{\begin{array}{l}
\frac{1}{N^{\prime}}\left|\frac{\sin \left(\pi f_{d} N^{\prime} T_{s}\right)}{\sin \left(\pi f_{d} T_{s}\right)}\right| \quad l=l j \\
0 \quad l \neq l j
\end{array}\right.
$$

where $N^{\prime}$ is the sample number. The detailed analysis of eqn (7) will be introduced by next section.

\section{THE ACQUisition Method UNDER LOW SNR}

Due to PCSS has a higher transmission rate than direct sequence spread spectrum system (DSSS) under the same bandwidth, which the reliability is reduced in low SNR. For 
this situation, the improved way is to accumulate some of the adjacent PN code periods [12].

Specifically as follows: In the first PN code period, all FFT correlation results of the $m$ frequency are denoted by

$$
\mathrm{Q}_{M^{*} N^{\prime}}^{m}(n)=\left[\mathrm{R}_{1}^{m}(n), \mathrm{R}_{2}^{m}(n), \cdots, \mathrm{R}_{M}^{m}(n)\right]^{\mathrm{T}}
$$

where $m=1,2, \cdots h, k=1,2, \cdots M$, and $\mathrm{R}_{k}^{m}(n)$ is the single channel FFT correlation results, then the $h \times N^{\prime}$ order FFT non-coherent accumulation results can be expressed as

$$
\mathrm{H}_{t}(n)=\left[\mathrm{R}_{\text {sum }}^{1}(n), \mathrm{R}_{\text {sum }}^{2}(n), \cdots \mathrm{R}_{\text {sum }}^{h}(n)\right]^{\mathrm{T}}
$$

Finally, the $K$ periods accumulation results can be represented as below

$$
\mathrm{T}_{\text {sum }}(n)=\sum_{t=1}^{K} \mathrm{H}_{t}(n)
$$

\section{SimUlation RESUlTS AND ANALYSIS}

In this paper, the following parameters are: (i) the number of orthogonal PN sequences $M$ is 16 , and the number of selected sequences $r$ is 3 , so the transmitted information is calculated by eqn (1): $k=12$ bits, (ii) a set of Gold sequence is chosen for the PN sequence, and the length of PN sequence is 128 , (iii) baseband data transmission rate is $4.8 \mathrm{kHz}$, (iv) carrier frequency is $204.8 \mathrm{kHz}$, and the sample rate for IF signal is $1.6384 \mathrm{MHz}$, (v) sweeping range is $\pm 10 \mathrm{kHz}$, and search step is $400 \mathrm{~Hz}$.

\section{A. PN Sequence Cross-Correlation on Suppressing Doppler Offset}

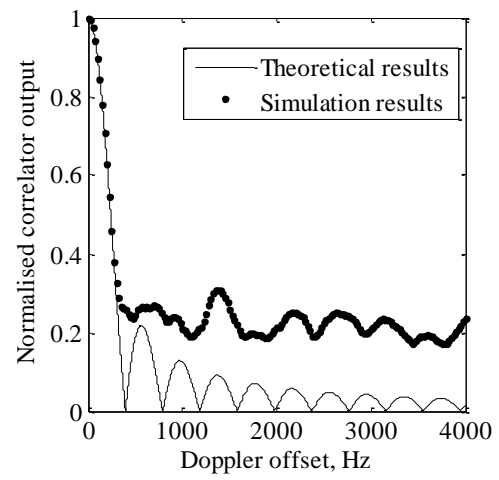

FIGURE V. FREQUENCY RESPONSE OF COMPLEX CORRELATOR

The frequency response of a complex correlator for a data rate of $400 \mathrm{~Hz}\left(N^{\prime}=4096, T_{s}=1 / 1.6384 \times 10^{6} \mathrm{~s}\right)$ is depicted in fig. 5. It can be seen that the normalized correlation peak is 1 with no Doppler offset, and that as the Doppler offset is increased the probability of detection is sharply decreased, especially the normalized correlation peak is 0 when $f_{d}=a / N^{\prime} T_{s}(a=1,2,3, \ldots)$. However, a irregular fluctuation appeared from the simulation values out of the mainlobe. This is because the PN sequences assumed are not completely orthogonal.

B. Impact of the Number of PN Code Period under Low SNR
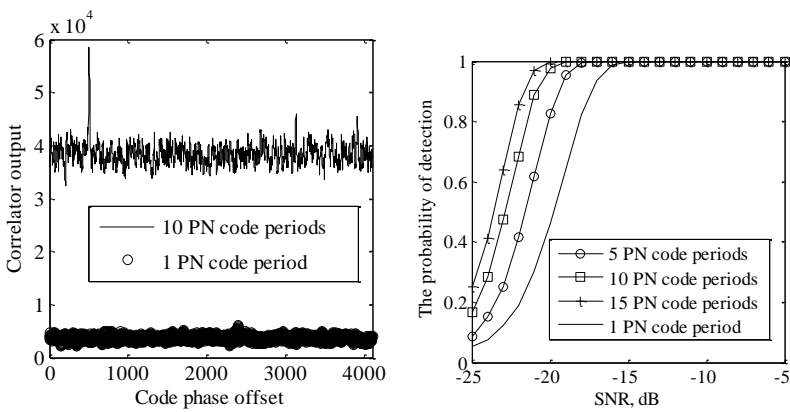

FIGURE VI. SNR=-20DB, THE CORRELATOR OUTPUT AND THE PROBABILITY OF DETECTION WITH DIFFERENT PN CODE PERIOD ACCUMULATED

Although $1 \mathrm{PN}$ code period correlation peak drowned in the noise which cannot be acquisition correctly, the acquisition correlation peak presented after $10 \mathrm{PN}$ code periods accumulated. It is illustrated that using only $1 \mathrm{PN}$ code period is not applicable under low SNR. As fig. 6 described that as the SNR increases the probability of detection rises. However, as the number of PN code periods is increased the computation complexity is also increased, so the selection of the number of PN code period should be integrated with computation and other parameters.

\section{CONCLUSIONS}

A time-frequency domain acquisition algorithm based on FFT has been proposed in this paper. Then, the principle of the algorithm is presented and the ability to suppress Doppler offset is analyzed, following by which the multiple correlation peak accumulation technology is introduced. The simulation results show that the new acquisition algorithm is able to complete the PCSS system acquisition, while a good performance of detection probability can be obtained by adjusting the number of PN code period in low SNR.

\section{ACKNOWLEDGEMENT}

This work was supported by National Natural Science Foundation of China (61101141 and 61401196), the Science and Technology on Information Transmission and Dissemination in Communication Networks Laboratory of China (KX132600013/ITD-U13006) and the Jiangsu Provincial Natural Science Foundation of China (grant No.: BK20140954).

\section{REFERENCES}

[1] ZHU J.K. \& MARUBAYASHI G., Properties and application of parallel combinatory ss communication system. IEEE Second International Symposium on Spread Spectrum Techniques and Applications, USA, pp. 227-230, 1992.

[2] SASAKI. S., ZHU J.K. \& MARUBAYASHI G., Performance of the parallel combinatory spread spectrum multiple access communication system with the error control technique. IEEE Second International Symposium on Spread Spectrum Techniques and Applications, Yokohama, Japan, pp. 159-162, 1992

[3] LIU M.D., LI H.L. \& GUO L.L., Research of soft decision modulations based on parallel combinatory spread spectrum system. Global Symposium on Millimeter Waves, Haerbin, pp. 507-511, 2012. 
[4] QI L., GUO L.L., \& JIANG X.F., Performance studies of parallel combinatory spread spectrum based on pulse modulation. Application Research of Computers, 28(6), pp. 2286-2288, 2011.

[5] GUO N., HONG F.M., \& LI L.M., An M-ary DS/SS communication system and the optimization of information-combinatory mapping. Acta Electronic Sinica, 24(4), pp. 50-53, 1996.

[6] GUO L.L., YI Q. \& LI B.M., Parallel combinatory spectrum communication system based on r-combinatory and the study of PN sequences. Radio Communications Technology, 33(4), pp. 25-27, 2007.

[7] GUO L.L., GUO L.L., \& JIANG X.F., Performance studies of UWB communication system based on parallel combinatory spread spectrum. WiCOM 2009, Beijing, China, pp. 1-4, 2009.

[8] HOU Y.F., \& HASE T., New OFDM structure with parallel combinatory code. IEEE Transactions on Consumer Electronics, 55(4), pp. 1854-1859, 2009.

[9] GUO L.L., LIU J.X., LI B.M. \& ZHANG W.C., Research on acquisition algorithm of combinatory Pseudo-Random codes in parallel combinatory spread spectrum system. Journal of Telemetry, Tracking and Command, 35(3), pp. 1-6, 2014.

[10] LIAN B.W., LIU H.H. \& MAO D.M., Fast Pseudo-Code acquisition method based on match filter and fast fourier transform and performance analysis. Measurement \& Control Technology, 28(1), pp. 20-23, 2009.

[11] XUE J., ZHAO Q. \& WU Z.J., Research on PN code fast acquisition algorithm based on FFT. Journal of Telemetry, Tracking and Command, 29(6), pp. 41-46, 2008.

[12] LI X. \& Zheng L.H., A PN code acquisition method and performance analysis in very low SNR. Microprocessors, (5), pp. 28-29, 2004. 